\title{
Enhanced Electromechanical Performance of Graphene Oxide-Nafion Nanocomposite Actuator
}

\author{
Wei Chang ${ }^{1,2}$, Huiqin $\mathrm{Lian}^{1, *}$, Yanou $\mathrm{Hu}^{1}$, Yueting $\mathrm{Li}^{1,2}$,Benzhe $\mathrm{Wu}^{1}$, Yupeng $\mathrm{Liu}^{1}$, Junlin Ma ${ }^{1}$, Junwei Ma ${ }^{1}, \mathrm{Jierui}$ Wang ${ }^{1}$ \\ ${ }^{1}$ Beijing Key Lab of Special Elastomer Composite Materials, College of Materials Science and Engineering, Beijing Institute of \\ Petrochemical Technology, Beijing 102617, China.hs \\ ${ }^{2}$ College of Materials Science and Engineering, Beijing University of Chemical Technology, Beijing 100029, China.hs.
}

\begin{abstract}
This topic involves graphene Nafion composite. Graphene is prepared by using hummer's method, thus the functional graphene. The functional Graphene-nafion composite has excellent electrical conductivity, thermal conductivity and mechanical properties. Therefore, it can be used as the preparation of high-strength conductive composites of ideal nano filler, and dispersed in the solution of graphene and Nafion, mixing to form a composite system, in addition to graphene with the composite function referring not only to excellent mechanical and electrical properties but also excellent processability, thus providing broader application field for composite materials.
\end{abstract}

\section{Introduction}

Ionic polymer-Nafion has the virtue of chemical stability and good mechanical property [1-2]. It has attracted much attention in the field of fuel cell, biosensor and artificial muscle. Therefore, it could be used in the field of biomimic area. An enhanced functional materials could made composite composed of nanomaterials and organic polymer being widely used in more fields.

With the development of new technology and new material, biomimetic materials was provided with a broader prospect. Meanwhile, artificial muscle material IPMC [3] is very suitable for the preparation of bionic artificial muscle material of the drive. It has been named in English as Ion - exchange Polymer Metal Composite (ion-exchange Polymer film Metal Composite materials). It has large deformation, good elasticity, and is very close to the biological characteristics of the muscle to a certain extent. Therefore, it is also known as artificial muscles. It is made up of the main chain with fluoride ion group closed period of the short side of the chain structure of the ion exchange membrane, and both sides of the electrode layers [4-5]. When applying excitation voltage electrodes on both sides of IPMC, it will produce a larger bending, generally to the anode, called direct phenomena. On the contrary, the action of external force when the IPMC bending deformation, the IPMC also can cause a voltage on the electrode on both sides[6], which is called the reverse phenomenon. In this way, IPMC forms a mechanical and electrical FGC system. Most research, home and abroad, has focused on how to improve the service life of IPMC, output displacement and output force, the study on preparation of IPMC and did research on its application in power devices [7-9].But there is also some scholars who devoted to find a kind of theoretical model that can simulate the IPM. By analyzing the model, they are able to understand its driving mechanism, in the future, to control force and output displacement of IPMC Thus they can provide more theoretical support and basis for the IPMC application in power devices.

Firstly, this topic is focused on the research of graphene - Nafion composite materials, graphene oxide prepared by Hummers method. Then functionalized graphene is prepared. Electric and thermal conductivity of graphene have outstanding performance, so that the preparation of high functional composites can be used as ideal nano filler. By dispersing in a solution, graphene can be mixed with Nafion to prepare nanocomposites. Graphene can be uniformly dispersed in the Nafion to prepare composites that have good mechanical, electrical responsiveness[10] and also the function of the preparation of graphene. Graphene that is structurally complete is made up of two-dimensional crystal which does not contain any key of benzene six-member ring combination. Thus it obtains high chemical stability and its surface is in inert state. And the interaction with other medium (such as solvent) is weak. At the same time, due to the strong vander Waals force among graphene pieces, graphene is more inclined to polymerize, making it difficult to soluble in water and common organic solvents, which will definitely limit the further research and application of graphene. On the other hand, on the surface of graphene oxide there exists a lot of oxygen containing functional groups, such as hydroxyl, carboxyl. These functional groups make modified graphene[11] possible. Graphene oxide is the starting point of large scale synthesis of graphene. It is also one of the most effective ways to realize the function of graphene. By applying the graphene oxide as a new type of packing to the preparation of functional polymer nanocomposites[12], we can improve nanometer composite material's comprehensive performances, such as force, heat, electricity, etc., thus making it widely used in environmental materials field that featured for its quality of pollution-free and low emission.

\section{Experimental and methods}

\subsection{Materials}

Tetrahydrofuran(THF,99wt\%purity),Sodiumnitrate(NaNO 3),PotassiumPermanganate(KMnO4),HydrogenPeroxide( $\mathrm{H} 2 \mathrm{O} 2)$ and Concentratedsulfuricacid $\left(\mathrm{H}_{2} \mathrm{SO}_{4}\right)$

were commercially available. N,N-Dimethylformamide (DMF, 99.9wt\% purity) was dehydrated at $100^{\circ} \mathrm{C}$ under vacuum for $24 \mathrm{~h}$ prior to use. Graphite (purity $>99 \%$ ) was provided by XFNANO MaterialsTech Co., Ltd. 
(Nanjing, China).

\subsection{Preparation of Graphene}

First, using the HUMMER method to obtain the Graphene Oxide(GO), then getting the Graphene through the microwave reduction reaction.

\subsection{Perparation of graphene-nafion}

The Nafion mixed with different ratio of Graphene cast film in the DMF solvent. There were three series of composite synthesized. Then in the drying oven bake for $12 \mathrm{~h}$ kept at $80{ }^{\circ} \mathrm{C}$.

\subsection{Measurements}

The structure of the GO and Graphene was analyzed by a Thermo Scientific Nicolet 6700-OMNI Smart Accessory Spectrum Fourier transform infrared (FTIR) spectrometer. Spectra were collected in the mid-infrared range from 5000 to $400 \mathrm{~cm}-1$ in the ART mode. X-ray Diffraction (XRD) spectra of the films were collected on Bruker D8 Focus. Data were collected between $10.93^{\circ}$ and $80.01^{\circ}$ with a step of $0.06^{\circ}$ and a dwell time of $1.5 \mathrm{~s}$. The morphology of films was examined using scanning electron microscopy (SEM, COXEM EM-20). The actuation performance was carried out using a homemade setup equipped with an electric balance. All the samples were cut into $30 \times 5 \mathrm{~mm}^{2}$ rectangles, including $5 \mathrm{~mm}$ of electrode contact area.

\section{Results and discussion}

\subsection{Structural and characterization}

The infrared spectra in Fig.1 show some typical functional groups of $\mathrm{GO}$ and Graphene. By Graphene Oxide (GO) absorption spectra, the methylene (-CH2) stretching vibration absorption peak at $3100 \mathrm{~cm}^{-1}$, the absorbance in the region near $1724 \mathrm{~cm}^{-1}$ is assigned to the stretching vibration of free $\mathrm{C}=\mathrm{O}$ groups, the key epoxy ether $(\mathrm{C}-\mathrm{O}-\mathrm{C})$ stretching vibration characteristic peak at $1220 \mathrm{~cm}^{-1}$. Above indicate that we get the graphene oxide successfully. On the contrary, there is nothing functional groups absorption peak in the graphene absorption spectra, it shows that grophene was prepared successfully.

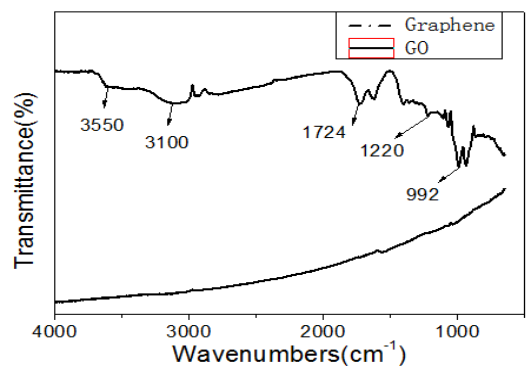

Figure1. FTIR spectra of Grephene and GO
The Graphite, Grahpene Oxide and Graphene was also studied by XRD in Fig.2. The X-ray diffractograms of the Graphite showed a sharpen diffraction peak reflections at $2 \theta=26.55^{\circ}$. It indicate that Graphite have higher lamella neat degree and crystallinity. At $2 \theta=10.25^{\circ}$ have a broad diffraction peak reflections in the X-ray diffractograms of the Graphene Oxide , with the increase of reaction time, the diffraction peak of Graphene Oxide become width and skewed in the direction of low Angle. This suggests that the graphite edge appeared all sorts of subtle oxygen-containing groups, surface slice layer structure disorder increases to a certain extent, lamella spacing increases. Near the diffraction peak at $2 \theta=26.55^{\circ}$ decreased obviously compared with Graphite. It shows that the level of flake Graphite layer stack reduce.

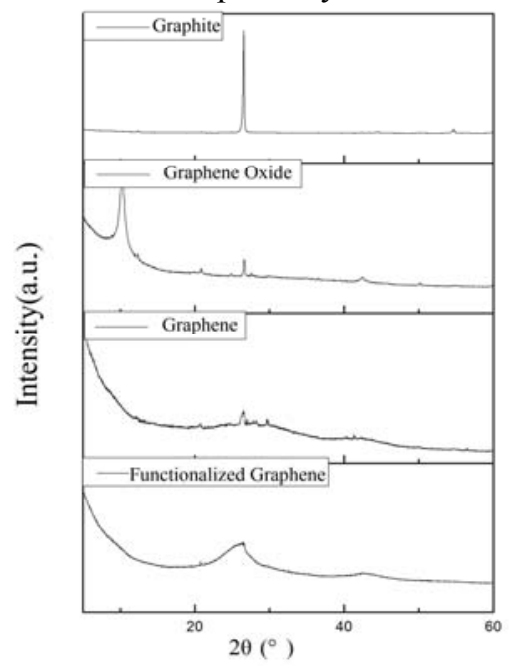

Figure 2. The XRD patterns of Graphite,Graphene Oxide, Graphene and functionalized Graphene

\subsection{SEM study}

The Fig.3 show the surface geometry analysis of the Graphene Oxide (A figure) and Graphene-nafion(B figure). From A figure can see Graphene surface in corrugated shape, single layer structure. The B figure is profile of composite membrane, it can be seen that even rough surface, Graphene and uniform dispersion in the Nafion matrix. The SEM of Graphene electrode(IPMC) in the Fig.4. It present that Graphene electrode layer evenly distributed on both sides of the membrane, the slice layer parallel to the membrane surface. 


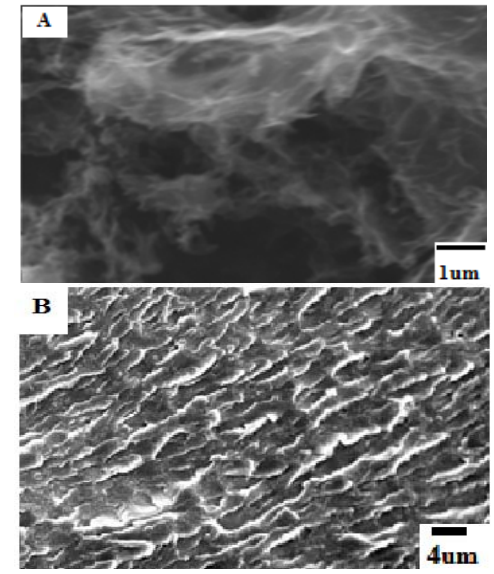

Figure3. The SEM images of cross section of A Graphene and B Composite

Table1. Composite material physical and mechanical performance

\begin{tabular}{lccccc}
\hline Sample & $\begin{array}{c}\text { The ratio } \\
\text { of } \\
\text { graphene } \\
(\%)\end{array}$ & $\begin{array}{c}\text { Swelling } \\
\text { rate }\end{array}$ & $\begin{array}{c}\text { Water-absorbing } \\
\text { quality }\end{array}$ & $\begin{array}{c}\text { Ion } \\
\text { exchange } \\
\text { property } \\
(\mathrm{mmol} / \mathrm{g})\end{array}$ & $\begin{array}{c}\text { Maximum } \\
\text { actuating } \\
\text { force }\end{array}$ \\
\hline Nafion & 0 & 9.67 & 25.88 & 0.41 & 2.3 \\
NfG1 & 1 & 9.72 & 26.70 & 0.49 & 4.0 \\
NfG2 & 2 & 9.89 & 25.97 & 0.43 & 2.8 \\
NfG5 & 5 & 9.80 & 27.45 & 0.38 & 2.8 \\
NfG7 & 7 & 9.77 & 25.89 & 0.44 & 2.1 \\
\hline
\end{tabular}

\subsection{Actuator property}

It shows the actuating property of composite membrane in Fig.5. It can be seen that the Nafion and composite membrane have good actuating. And the maximum actuating force of NfG1 is double of composite's. From the table1,it shows that with the increase of functional graphite content, the actuating force reduced, because the functionalized Graphene itself does not contain ionic groups, which affect the number of ion migration.

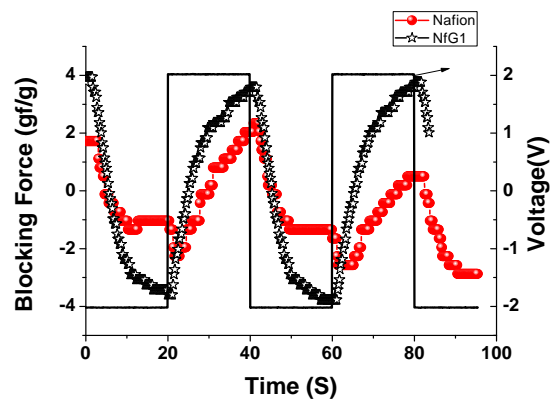

Figure 5. The Actuator property of composite materials

\section{Conclusion}

A novel structure of graphene-Nafion composite was proposed. It has good mechanical and electrical responsiveness. Successful Graphene Oxide was prepared by Hummers method. Thanks to a large amount of oxygen-containing groups contained in the graphene, which makes the layer spacing bigger, the preparation of functionalized graphene and the optimization and improvement of its preparation technology can be possible.

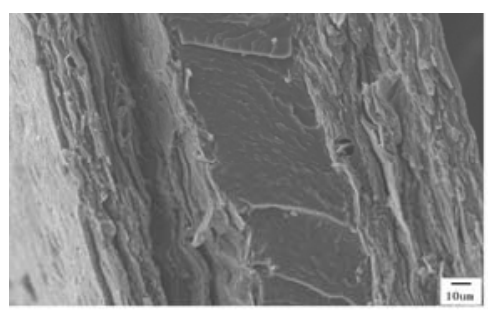

Fig.4. The SEM of Graphene electrode

\subsection{Property test}

The property of different ratio of Graphene used for the composite is listed in Table 1.

\section{Acknowledgements}

This work was supported by the Natural Science Foundation of China (NSFC, Nos. 51063009, 21271031, 51203012), the Beijing Natural Science Foundation of China (Nos. 2132009, 2122015), undergraduate Research Training Program of Beijing (2015J00002) and the project about the promotion of innovation in Beijing universities funded by Beijing Municipal commission of education of China.

\section{References}

[1] J.N. Rodriguez, F.J. Clubb, T.S. Wilson, M.W. Miller, T.W. Fossum, J. Hartman, E. Tuzun. J. Biomed. Mater. Res., Part A. 102A (2014) 1231-1242.

[2] C. Ohm, M. Brehmer, R. Zentel. Adv. Mater. (Weinheim, Ger.). 22 (2010) 3366-3387.

[3] Punning A, KruusmaaM, AablooA. [J]. Sensor and Actuators A: Physical, 2007, 133(1): 200-209.

[4] Lian Y, Liu Y, Jiang T, Shu J, Lian H, Cao M (2010). J Phys Chem C 114(21):9659-9663.

5. Sun G, Pan Y, Zhan Z, Zheng L, Lu J, John HL et al (2011) J Phys Chem C 115:23741-23744.

6. Cai D, Yusoh K, Song M. Nanotechnology. 2009;20:085712. 
[5] 7. Hou,Li Jun,Zhang, et al. [J] Carbon, 2001,39: 915-920.

[6] 8. K.S. Novoselov, A.K. Geim, S.V. Morozov, D. Jiang, Y. Zhang, S.V. Dubonos, et al..Science 306 (2004) 666-669.

9. S. Park, R.S. Ruoff. Nat.Nanotechnol. 4 (2009) 217-224.

[7] 10.Yang N, Zoski CG. Langmuir 2006;22(25):10338-47.

[8] 11. Yang L, Phua SL, Toh CL, Zhang L, Ling H, Chang M, et al. RSC Adv 2013;3(18):6377.

[9] 12. N.G. Sahoo, H.K.F. Cheng, L. Li, S.H. Chan, Z. Judeh, J.H. Zhao, Specific. Adv. Funct. Mater. 19 (2009) 3962-3971. 